\title{
Caracterización de recubrimientos DLC/Si bajo la influencia de un fluido biológico simulado
}

\author{
Characterization of DLC/Si coatings under the influence of a \\ simulated biological fluid
}

\section{Caracterização de recobrimentos DLC/Si sob a influência de um fluído biológico simulado}

Fecha de recepción: 27 de septiembre de 2015

Fecha de aprobación: 06 de diciembre de 2015
William Vargas*

José Luis Caballero-Gómez**

Willian Arnulfo Aperador-Chaparro***

\section{Resumen}

Uno de los materiales más usados como biomaterial es el acero 316LVM; sin embargo, presenta complicaciones al trabajarlo como reemplazo permanente, debido a que libera iones metálicos a los tejidos, generando aumento en el número de intervenciones que deben realizarse; ante esto, se estudia cómo mejorar el comportamiento de materiales convencionales mediante recubrimientos que elevan sus propiedades mecánicas y anticorrosivas, incrementando su vida útil. Se evaluó el comportamiento ante el fenómeno de micro-abrasión-corrosión de recubrimientos DLC/Si depositados mediante la técnica de deposición química de vapor asistida por plasma en contacto con solución de Ringer, simulando un ambiente biológico. Los resultados indican que el recubrimiento experimenta un aumento en el volumen de desgaste cuando se encuentra sometido al mecanismo de micro-abrasión-corrosión en relación con la prueba de solo microabrasión, además, la pérdida más significativa se encuentra en el sustrato sin recubrimiento; esto demuestra el efecto protector del recubrimiento.

Palabras clave: Recubrimientos, Microabrasión, Fluido biológico.

\section{Abstract}

One of the metals most used as biomaterial is the 316LVM steel. Nevertheless, it presents complications when used as permanent implant, due to the metallic ion release to the surrounding tissues, leading to higher number of

\footnotetext{
* M.Sc. Servicio Nacional de Aprendizaje - SENA - Centro de Diseño y Metrología (Bogotá-Distrito Capital, Colombia).wupntco@misena.edu.co.

** M.Sc. Universidad Militar Nueva Granada (Bogotá-Distrito Capital, Colombia).

*** Ph.D. Universidad Militar Nueva Granada (Bogotá-Distrito Capital, Colombia). william.aperador@unimilitar.edu.co.
} 
interventions that the patients must undergo. Given this problem, it was studied how to improve the conventional materials coatings behavior that can raise their mechanical and anti-corrosive properties, increasing their service life. This behavior was evaluated facing the micro-abrasion-corrosion of DLC/Si, coatings phenomena, deposited by using the assisted chemical vapor deposition by plasma in contact with the Ringer solution, simulating a biological environment. The results indicate that the coating experiences an increase in the wear volume, when is subjected to the micro-abrasion-corrosion mechanism, in comparison with the micro-abrasion test. Moreover, the most significant loss is found in the substrate without coating, which demonstrates the protective effect of the coating.

Key words: Coatings, Micro-Abrasion, Biological fluid.

\section{Resumo}

Um dos materiais mais usados como biomaterial é o aço 316LVM; porém, apresenta complicações ao trabalhálo como substituto permanente, devido a que libera íons metálicos aos tecidos, gerando aumento no número de intervenções que devem realizar-se; ante isto, estuda-se como melhorar o comportamento de materiais convencionais mediante recobrimentos que elevam suas propriedades mecânicas e anticorrosivas, incrementando sua vida útil. Foi avaliado o comportamento ante o fenômeno de micro-abrasão-corrosão de recobrimentos DLC/Si depositados mediante a técnica de deposição química de vapor assistida por plasma em contato com a solução de Ringer, simulando um ambiente biológico. Os resultados indicam que o recobrimento experimenta um aumento no volume de desgaste quando encontra-se submetido ao mecanismo de micro-abrasão-corrosão em relação com a prova somente de microabrasão, além disso, a perda mais significativa encontra-se no substrato sem recobrimento; isto demonstra o efeito protetor do recobrimento.

Palavras chave: Recobrimentos, Microabrasão, Fluído biológico.

Cómo citar este artículo:

[1] W. Vargas, J. L. Caballero-Gómez \& W. A. Aperador-Chaparro, "Caracterización de recubrimientos DLC/ Si bajo la influencia de un fluido biológico simulado”, Fac. Ing., vol. 25 (41), pp. 7-19, ene.-abr. 2016. 


\section{INTRODUCCIÓN}

El incremento de la vida útil de los implantes biomédicos es hoy un área de desarrollo, ya que los materiales utilizados convencionalmente presentan inconvenientes en su desempeño a largo plazo. Por ejemplo, en un reemplazo total de cadera, la cabeza femoral metálica se desliza contra una copa de polietileno, generando partículas poliméricas que pueden provocar reacciones inflamatorias que causan la osteólisis (reabsorción ósea); además, la migración de iones metálicos hacia los tejidos circundantes aumenta el riesgo de aparición de tumores y de daños en el ADN [1-3]. Debido a estas complicaciones, la frecuencia con la que se realiza una segunda o tercera intervención es alta, disminuyendo la calidad de vida de los pacientes y aumentando los costos de la salud; de allí, la demanda de biomateriales más duraderos [4, 5].

El acero inoxidable es una aleación de hierro, carbono y, al menos, un $11 \%$ de cromo; este cromo es responsable de que la aleación sea más resistente a la corrosión que un metal común [6]. Las propiedades mecánicas del acero y su gran resistencia a la corrosión lo hacen adecuado para ser utilizado en ambientes agresivos, como intercambiadores de calor, equipos para la industria petrolera o láctea y el tratamiento de aguas residuales, y para implantes biomédicos, entre otras, [7, 8]. A pesar de esa gran resistencia a la corrosión, estos aceros inoxidables son susceptibles de sufrir corrosión por picadura en entornos con cloruro [14], razón por la cual se hace necesario investigar alrededor de un tipo de recubrimiento superficial que busque mejorar la resistencia a la abrasión y la corrosión.

Durante décadas, los recubrimientos superficiales han sido investigados, gracias a sus ventajas en cuanto a la protección y modificación de superficies [9-11]; según varias investigaciones, el recubrimiento de DLC (Diamond Like Carbon, Carbono tipo diamante) se caracteriza por su buena resistencia a la fricción, generalmente bajo condición de fricción en seco [12], y por tener alta adherencia en diferentes sustratos [13-14]. Los recubrimientos sobre implantes han revelado que el deslizamiento y la abrasión son los mecanismos de desgaste predominantes [15]; pero encontrar un recubrimiento óptimo para reducir la fricción es enormemente dispendioso, debido a la enorme cantidad de recubrimientos disponibles; por esta razón se espera que métodos sencillos como el
Ball Cratering o la microabrasión puedan ayudar a evaluar las propiedades del recubrimiento y servir como herramientas de preselección, con el fin de reducir el número de recubrimientos candidatos y así escoger la mejor opción [16].

La microabrasión consiste en una mezcla abrasiva que cae sobre una bola de acero giratorio que se encuentra presionando la muestra por estudiar. La influencia de medios corrosivos y la eliminación del material mediante abrasión-corrosión aún se encuentran en etapas de investigación [4]. Adicionalmente, el método de microabrasión permite evaluar las muestras en un medio electrolítico para estudiar los fenómenos corrosivos; por tal razón, se presenta como técnica para determinar los mecanismos de desgaste que se presentan en el acero inoxidable 316LVM cuando tiene un recubrimiento de DLC.

Una de las alternativas para mejorar el comportamiento de reemplazos articulares son los recubrimientos duros, pues en aplicaciones técnicas, como en herramientas de corte y mecanizado, elevan las propiedades del sustrato, mejorando su resistencia al desgaste y a la corrosión. Dentro de estos recubrimientos se encuentran los de carbono tipo diamante (DLC), que es un material con propiedades mecánicas, tribológicas y biológicas sobresalientes [17].

En el presente trabajo se realizaron pruebas de microabrasión sobre muestras de recubrimientos DLC/Si depositados sobre sustratos de acero AISI 316 LVM contra una esfera de hueso (cóndilo de bovino) en contacto con lactato de Ringer con micropartículas de alúmina en suspensión, para simular condiciones biológicas de desgaste.

\section{Materiales Y MÉtodos}

Se emplearon muestras de acero inoxidable AISI $316 \mathrm{LVM}$ de $20 \mathrm{~mm} \mathrm{X} 30 \mathrm{~mm}$; estos sustratos fueron cortados con el proceso de electroerosión de corte por hilo a una velocidad de corte de $5 \mathrm{~mm} / \mathrm{min}$; después del corte se pulieron con papel de carburo de silicio de $80,120,160,220,240,360,500,1000$ y 1200 , y por último se usó un paño micro cloth y solución abrasiva de alúmina de $0.03 \mu \mathrm{m}$. La deposición de la película de carbón tipo diamante (DLC) se realizó mediante la técnica de deposición química de vapor optimizada por plasma (PECVD). Los sustratos de AISI 316LVM fueron colocadas en un cátodo refrigerado por agua 
suministrada por una fuente DC pulsada bipolar asimétrica. Posteriormente se generó vacío, para luego purgar el sistema y llevar a cabo una segunda limpieza con plasma de Argón durante 30 minutos, con el objetivo de remover cualquier capa de óxido superficial, y nuevamente la cámara es sometida a un alto vacío de $10^{-6}$ Torr. Para mejorar la adhesión de la película de DLC se generaron capas intermedias de Silicio amorfo fino $(\sim 100 \mathrm{~nm})$, utilizando silano como precursor durante 15 minutos. Posteriormente se permite la entrada del acetileno a una presión que se encuentra en la franja de $2,5 \times 10^{-3}$ Torr; la forma de onda consistió en un pulso positivo fijo de $30 \mathrm{~V}$, seguido por un pulso negativo variable que oscila entre -250 hasta $-700 \mathrm{~V}$; en el pulso se utilizó una frecuencia de $20 \mathrm{kHz}$; las películas se depositaron con metano a una presión de $10 \mathrm{~Pa}$ a $10 \mathrm{sccm}$ de flujo.
Los ensayos de micro-abrasión-corrosión fueron llevados a cabo en un equipo que fue modificado para realizar mediciones de la actividad electroquímica y controlar el potencial aplicado durante la prueba de desgaste micro-abrasivo usando un potenciostatogalvanostato. La Fig. 1 muestra el diagrama esquemático del equipo adecuado; para mediciones de la tasa de corrosión se tiene una configuración de tres electrodos: las muestras actúan como electrodo de trabajo; un alambre de platino, como contraelectrodo, $y$ un electrodo de $\mathrm{Ag} / \mathrm{AgCl}$, contenido en un tubo capilar, como el electrodo de referencia; los electrodos se encuentran inmersos en una solución fisiológica que sirva como electrolito.

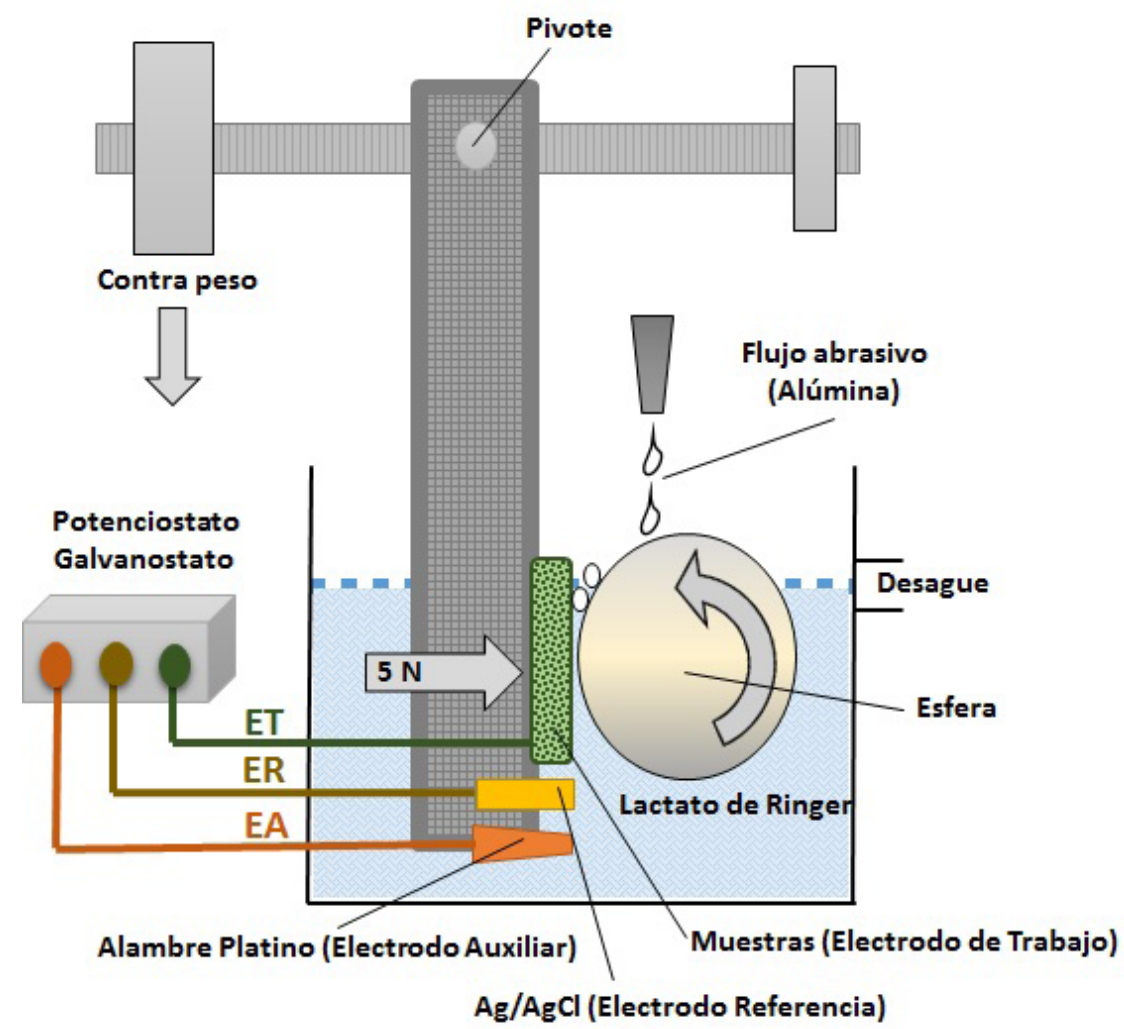

Fig. 1. Esquema de equipo de microabrasión configurado para pruebas electroquímicas.

El recubrimiento fue caracterizado mediante difracción de rayos X (DRX). El arreglo experimental corresponde a Goniómetro PW3050/60 ( $(\theta / \theta)$, manejado bajo un sistema XPERT-PRO, usando una radiación monocromática de $\mathrm{Cu} \mathrm{K \alpha} 1,54 \AA$, operado a $40 \mathrm{kV}$ y $40 \mathrm{~mA}$ bajo condiciones de temperatura de 25 ${ }^{\circ} \mathrm{C}$. El barrido sobre la superficie fue realizado desde
$2 \theta=20,01^{\circ}$ hasta $2 \theta=99,99^{\circ}$, con un paso $2 \theta=0,02^{\circ}$ a un tiempo de barrido de 1 segundo, utilizando la base de datos de difracción del equipo; además, se empleó el programa de análisis MAUD, que se basa en el método Rietveld; este método consiste en ajustar un diagrama teórico para que coincida en su totalidad con el observado. El análisis topográfico de las superficies 
se elaboró mediante el microscopio de fuerza atómica Nanosurf, en el que se adaptó una punta de contacto y se ajustó el láser que forma parte del sistema de retroalimentación; el barrido que se analizó tenía un área de aproximadamente $46 \mu \mathrm{m}^{2}$, y para la obtención de los datos se utilizó el software SPIPTM.

\section{Resultados}

\section{A. Difracción de rayos $X$}

En la Fig. 2 se muestran los patrones de difracción de rayos X de la película de carbono DLC depositados sobre acero $316 \mathrm{LVM}$, con el objetivo de identificar la fase de carbono. El pico a $43,4^{\circ}$ podría ser asignado al (111) espaciado reticular de diamante cúbico, que puede ser considerado la presencia de dominios de carbono sp3 ordenado locales en la matriz de carbono amorfo. A partir de los patrones de difracción de rayos $\mathrm{X}$ en la Fig. 2 se puede también observar que el pico de difracción a $43,4^{\circ}$ no tiene ninguna diferencia obvia para la película de carbono puro en la matriz de carbono amorfo correspondiente al DLC, la indexación correspondiente a un mineral con un sistema cristalino cúbico, con un número de PDF -01-075-0220 (PDF: Powder Difracction File), con un parámetro de red de $3.7670 \AA$ Å ángulos de $90^{\circ}$.

\section{(111)}

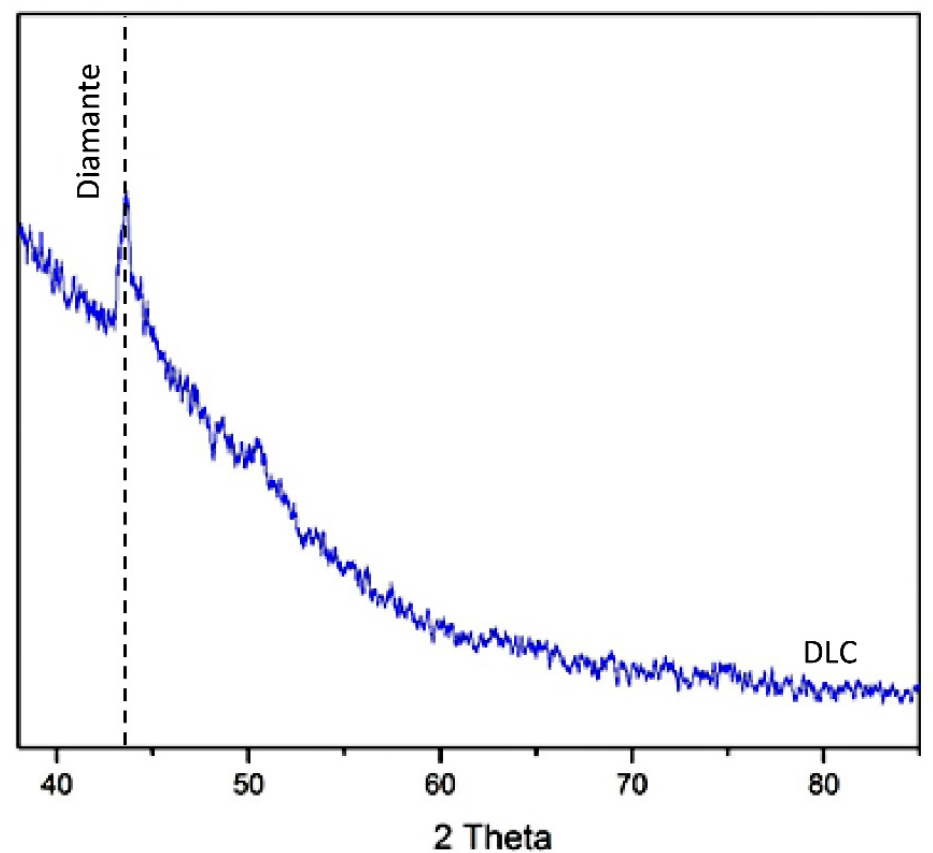

Fig. 2. Difractograma del recubrimiento DLC sobre el sustrato de acero 316 LVM.

\section{B. Estimación del desgaste microabrasivo}

Para estimar el desgaste se usó un modelo matemático que relaciona las dimensiones de la huella generada por el mecanismo con un volumen y una constante de desgaste; donde el volumen de desgaste $V$ está dado por la relación entre diámetro de la huella $b$ y el radio del pin de hueso con el que se realizó el desgaste $R$ $(12,5 \mathrm{~mm})$, como se observa en (1).

$$
V=\frac{\pi b^{4}}{64 R}
$$

Con el volumen de desgaste calculado se estimó una constante de desgastes mediante (2), donde $S$ es la distancia lineal recorrida, que fue $157,08 \mathrm{~m}, \mathrm{y} N$, la carga normal aplicada, que fue $5 \mathrm{~N}$.

$$
K=\frac{V}{S N}
$$

Por lo tanto, después de cada prueba fueron medidas las huellas de desgaste mediante microscopia óptica, como se observa en la Fig. 3. 


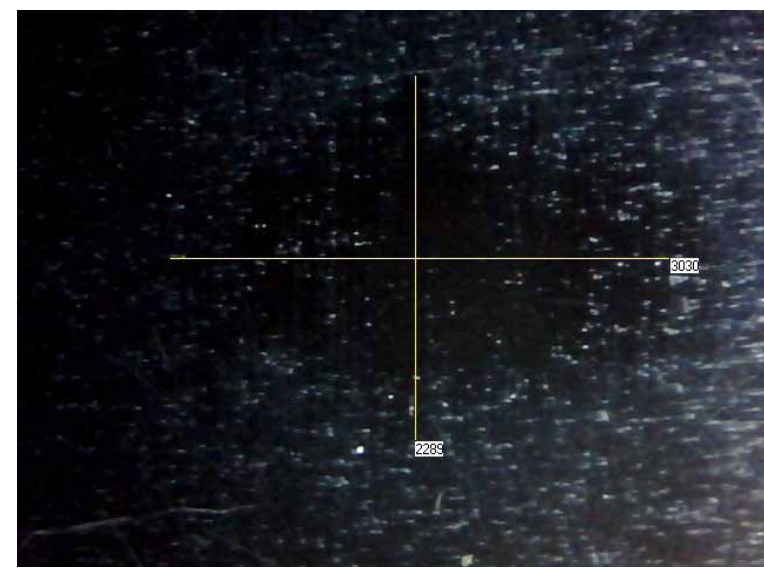

Fig. 3. Medición de la huella de desgaste del recubrimiento DLC/Si mediante microscopia óptica.
A partir de las mediciones se obtuvieron los volúmenes y constantes de desgaste para cada caso, como se muestra en la Tabla I. Se obtuvo que el recubrimiento muestra un mejor comportamiento que el sustrato; aun con la presencia de los fenómenos corrosivos, se observa que el recubrimiento exhibe volúmenes de desgaste menores que el acero 316 LVM. Por otro parte, tanto el sustrato como el recubrimiento experimentan un aumento en el volumen de desgaste cuando se encuentran sometidos al mecanismo de micro-abrasión-corrosión, donde el incremento es más significativo para el sustrato.

\section{TABLA I}

DATOS DE VOLÚMENES Y CONSTANTES DE DESGASTE

\begin{tabular}{|c|c|c|}
\hline Muestras & $\begin{array}{c}\text { Volumen de desgaste } \\
{\left[\mathrm{mm}^{3}\right]}\end{array}$ & $\begin{array}{c}\text { Constante de desgaste } \\
{\left[\mathrm{mm}^{3} / \mathbf{N ~ m ]}\right.}\end{array}$ \\
\hline $\begin{array}{l}316 \text { LVM } \\
\text { (Micro-abrasión) }\end{array}$ & 8,28 & $1,05 \times 10^{-2}$ \\
\hline $\begin{array}{l}316 \text { LVM } \\
\text { (Micro-abrasión-corrosión) }\end{array}$ & 14,91 & $1,90 \times 10^{-2}$ \\
\hline $\begin{array}{l}\text { DLC/Si } \\
\text { (Micro-abrasión) }\end{array}$ & 3,14 & $4 \times 10^{-3}$ \\
\hline $\begin{array}{l}\mathrm{DLC} / \mathrm{Si} \\
\text { (Micro-abrasión-corrosión) }\end{array}$ & 5,77 & $7,35 \times 10^{-3}$ \\
\hline
\end{tabular}

\section{Espectroscopia de impedancia electroquímica}

(EIS)

En la Fig. 4, se observa el diagrama de Nyquist; en él se compara la respuesta electroquímica del acero 316LVM y del recubrimiento de DLC; la curva generada indica el adecuado desempeño logrado por el recubrimiento, pues aumenta en más de tres veces la resistencia total del sistema, debido al efecto de película protectora, cuya porosidad y rugosidad están en el orden de los nanómetros, lo que genera una disminución de la dispersión de la frecuencia. La estructura obtenida genera un excelente comportamiento, como consecuencia de la interfaz que existe entre el sustrato y la capa de DLC, generada por el silicio, que permite una transición suave entre la unión de los dos sistemas. Por lo tanto, con un espesor de $3 \mu \mathrm{m}$ de la capa de revestimiento de unión, el recubrimiento se analiza como un solo elemento; por lo que un esquema de corriente alterna es correspondiente al circuito equivalente en donde se tiene una resistencia a la polarización que indica el desempeño de la capa protectora en paralelo con un elemento de fase constante, evidenciando la adecuada adherencia del recubrimiento al acero. 


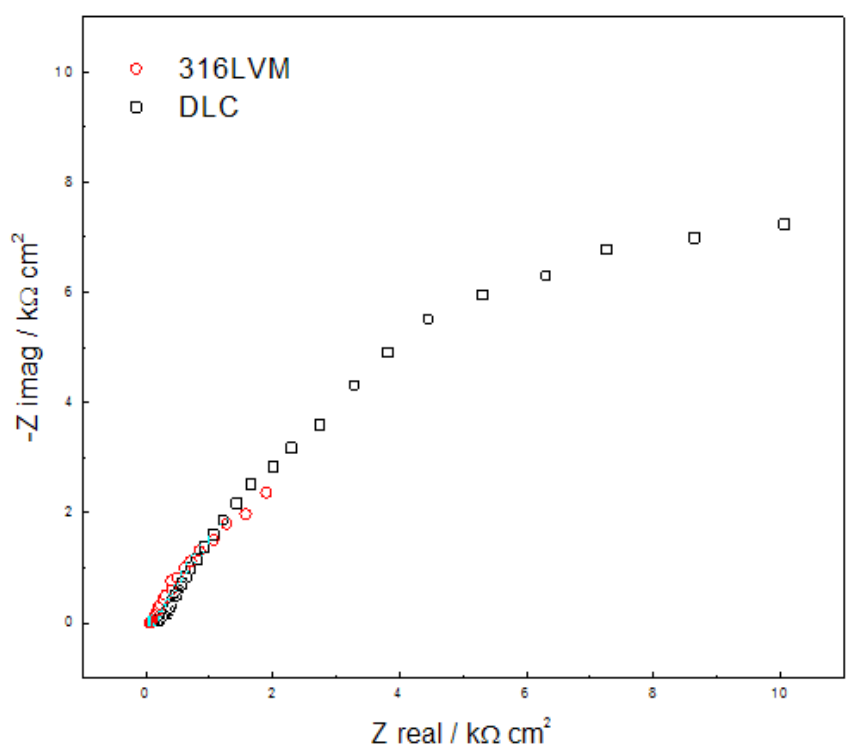

Fig. 4. Resultados espectroscopia de impedancia electroquímica de sustrato 316LVM sin recubrimiento y con recubrimiento DLC/Si.

En la Tabla II se presentan los valores de los ajustes teóricos realizados mediante un circuito equivalente que corresponde a una celda de Randles que tiene la resistencia a la corrosión en paralelo con el elemento de fase constante que cumple la función de capacitor y que tiene en cuenta la rugosidad del material.

\section{TABLA II}

PARÁmETROS DE LA PRUEBA EIS SOBRE SUSTRATO DE 316LVM

\begin{tabular}{|c|c|c|}
\hline Parámetro & Valor & Unidades \\
\hline Resistencia a la solución & 83,12 & Ohms \\
\hline Resistencia a la polarización & 8,46 & Kohms \\
\hline $\begin{array}{c}\text { Elemento de fase constante de interfaz } \\
\text { sustrato solución }\end{array}$ & $4,53 \times 10^{-7}$ & $\mathrm{~S}^{*} \mathrm{~s}^{\wedge} \mathrm{a}$ \\
\hline $\begin{array}{c}\text { Parámetro ajustable de carácter empírico } \\
/ \mathrm{n}\end{array}$ & 0,842 & \\
\hline
\end{tabular}

La Tabla III contiene el análisis de la película que actúa como recubrimiento; se puede determinar que la película contiene los mismos elementos que el acero 316LVM, pero con diferentes valores, ya que la interacción con el sistema es diferente por la generación de una nueva interfaz, correspondiente entre acero y silicio; adicional a esto existe un aumento en el nuevo elemento que corresponde al DLC; el valor de resistencia a la polarización, de 18,21 kohms, corresponde al efecto protector que genera el sistema debido a bajo coeficiente de fricción y buena resistencia a la corrosión. 


\section{TABla III}

PARÁMETROS DE LA PRUEBA EIS SOBRE SUSTRATO DE 316LVM CON RECUBRIMIENTO DLC/Si

\begin{tabular}{|c|c|c|}
\hline Parámetro & Valor & Unidades \\
\hline Resistencia a la solución & 126 & Ohms \\
\hline Resistencia a la polarización & 6,5 & $\mathrm{kohms}$ \\
\hline Resistencia a la corrosión & 18,21 & $\mathrm{kohms}$ \\
\hline $\begin{array}{c}\text { Elemento de fase constante de } \\
\text { interfaz recubrimiento solución }\end{array}$ & $5,34 \times 10^{-8}$ & $\mathrm{~S}^{\wedge} \mathrm{s}^{\mathrm{a}} \mathrm{a}$ \\
\hline $\begin{array}{c}\text { Parámetro ajustable de carácter } \\
\text { empírico / }\end{array}$ & 0,76 & $\mathrm{~S}^{*} \mathrm{~s}^{\wedge} \mathrm{a}$ \\
\hline $\begin{array}{c}\text { Elemento de fase constante de } \\
\text { interfaz sustrato recubrimiento }\end{array}$ & $3,54 \times 10^{-9}$ & \\
\hline $\begin{array}{c}\text { Parámetro ajustable de carácter } \\
\text { empírico / m }\end{array}$ & 0,89 & \\
\hline
\end{tabular}

\section{Curvas potenciodinámicasTafel}

En la Fig. 5 se detallan las curvas potenciodinámicas correspondientes al acero AISI 316LVM y al recubrimiento DLC. El acero tiene un potencial de corrosión hacia una zona catódica, lo cual genera un bajo desempeño electroquímico; la región anódica indica una disolución acelerada, debido a que la corriente de corrosión tiene un incremento de forma espontánea, con pequeños aumentos de potencial; esto evidencia el valor que se adquiere en la corriente y velocidad de corrosión. El recubrimiento tiene un mejor desempeño, debido a que su potencial de corrosión, en comparación con el sustrato, muestra un desplazamiento hacia la región anódica, genera una inercia química e incrementa su resistencia a la corrosión; la curva potenciodinámica genera un aumento gradual de la corriente de corrosión en las zonas tanto anódicas como catódicas, lo que representa un bajo valor de corriente y velocidad de corrosión, valores expuestos en la Tabla IV, corroborando la disminución del efecto de degradación [18]. 


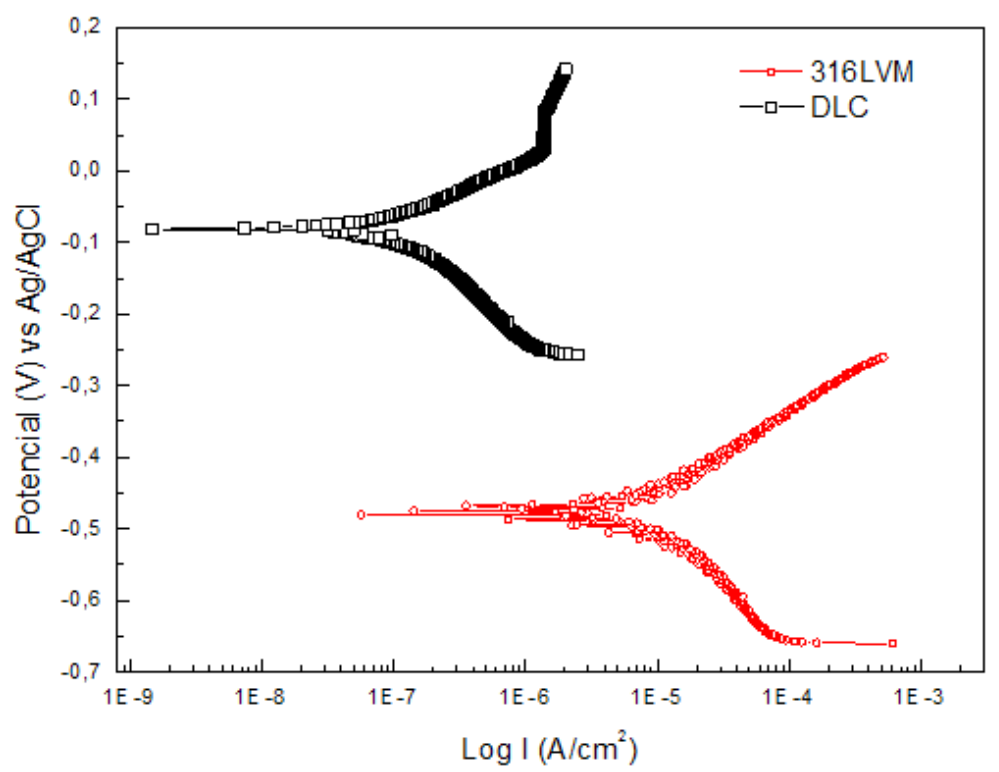

Fig. 5. Curvas Potenciodinámicas Tafel de sustrato 316LVM vs. recubrimiento DLC/Si.

TABLA IV

RESULTADOS DE CURVAS POTENCIODINÁMICAS TAFEL

\begin{tabular}{|c|c|c|c|}
\hline Parámetro & $\begin{array}{c}\text { Potencial de corrosión } \\
/ \mathbf{m V}\end{array}$ & $\begin{array}{c}\text { Corriente de corrosión / } \\
\mathbf{A - \mathbf { c m } ^ { - 2 }}\end{array}$ & $\begin{array}{c}\text { Velocidad de } \\
\text { corrosión / } \mathbf{m p y}\end{array}$ \\
\hline 316LVM & -478 & $1,02 \times 10^{-5}$ & 4,66 \\
\hline DLC & -81 & $1,56 \times 10^{-7}$ & 0,071 \\
\hline
\end{tabular}

\section{E. Microscopía de fuerza atómica (AFM)}

Para completar la caracterización morfológica de las muestras de sustrato 316LVM y sustratorecubrimiento de DLC se implementó un análisis mediante un microscopio de fuerza atómica. En cada muestra se analizó un área de 46,2 $\mu \mathrm{m}$ x 46,2 $\mu \mathrm{m}$ en modo contacto, con lo cual se obtuvo la rugosidad promedio para cada ensayo; a continuación se observa la rugosidad de cada muestra con relación a cada ensayo realizado (Tabla V).

\section{Tabla V}

DATOS DE RUGOSIDAD OBTENIDA EN CADA ENSAYO

\begin{tabular}{|c|c|c|}
\hline Probeta & Ensayo Realizado & Rugosidad Promedio $(\boldsymbol{\mu m})$ \\
\hline DLC Base & - & 13,74 \\
\hline 0.3-DLCh & Corrosión-Desgaste & 9,7677 \\
\hline 0.1-DLC & Desgaste & 9,677 \\
\hline 316L Base & - & 11,427 \\
\hline 316L Base & Corrosión-Desgaste & 66,533 \\
\hline 316L Base & Desgaste & 60,788 \\
\hline
\end{tabular}


Además de esto se generó una imagen 3D de cada superficie analizada por medio del software SPIP, con el fin de comparar la superficie después de cada ensayo. En la Fig. 6 se observan las imágenes obtenidas para el acero $316 \mathrm{LVM}$ con los respectivos ensayos realizados.

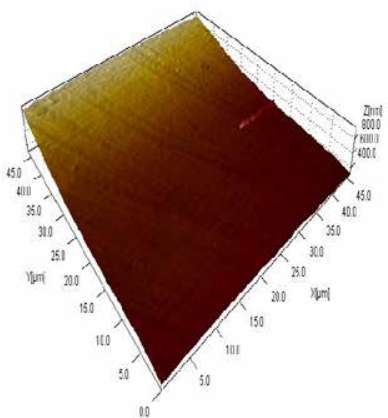

(a)

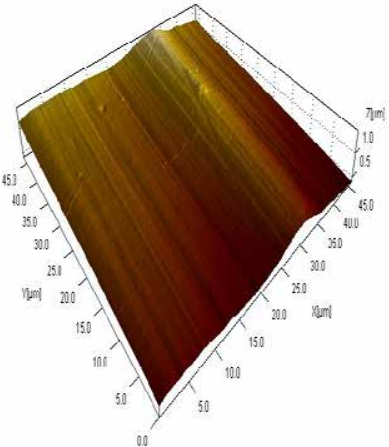

(b)

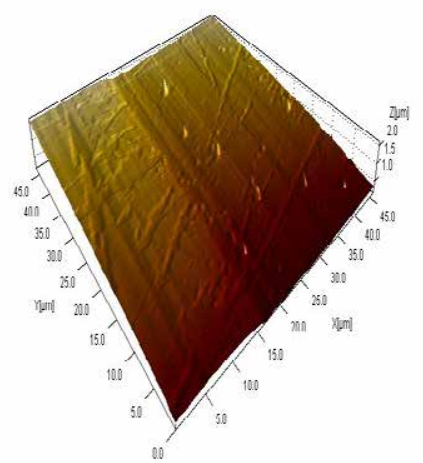

(c)

Fig. 6. Microscopía de fuerza atómica: a) Acero base, b) Acero sometido a corrosión, c) Acero sometido a desgaste.

En comparación, se observan los tres sistemas obtenidos del acero base, en los cuales, inicialmente, se tiene una rugosidad de $11,427 \mu \mathrm{m}$, considerada baja; pero al aplicar los fenómenos de corrosión y desgaste se observa cómo las rugosidades obtenidas se elevan de manera considerable, a $66,533 \mu \mathrm{m}$ y 60,788 $\mu \mathrm{m}$ para las muestras sometidas a corrosión-desgaste y desgaste, respectivamente. Este efecto de aumento de la rugosidad puede atribuirse a la degradación superficial que sufre el material, tanto en desgaste como en corrosión, con lo cual se generan más crestas $\mathrm{y}$ valles, aumentando la rugosidad superficial.
En la Fig. 7 se observan las imágenes obtenidas para las muestras de sustrato-recubrimiento de DLC; se puede notar que el recubrimiento base tiene una rugosidad de $13,74 \mu \mathrm{m}$, pero que al aplicar los fenómenos de desgaste y corrosión la rugosidad media del área analizada se disminuye para los dos casos de estudio. Los resultados obtenidos son una rugosidad de 9,7677 $\mu \mathrm{m}$ (corrosión-desgaste) y 9,677 $\mu \mathrm{m}$ (desgaste), y a partir de estos resultados se puede inferir que en los dos ensayos se generó una eliminación de crestas en la superficie del material sin presentar la eliminación total de ellas, con lo cual se resalta la protección del recubrimiento hacia el sistema. 


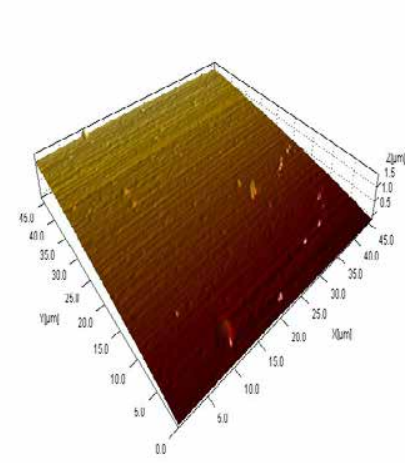

(a)

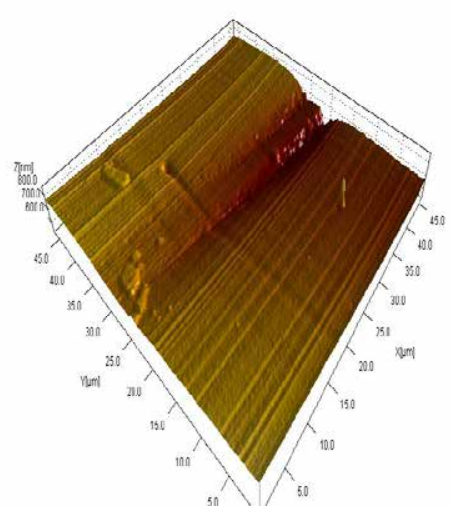

(b)

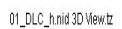

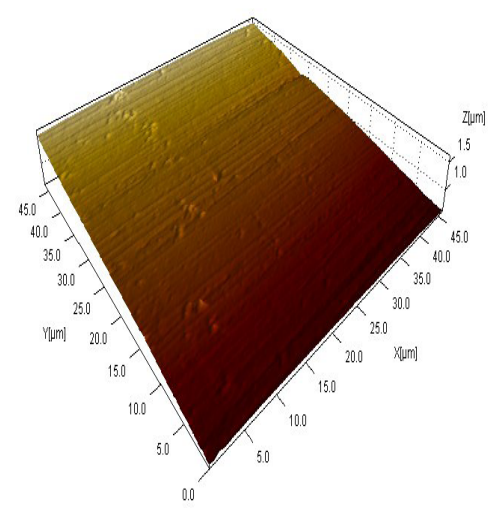

(c)

Fig. 7. Microscopía de fuerza atómica: a) DLC base, b) DLC sometido corrosión, c) DLC sometido a desgaste.

Comparando los dos sistemas, de acero y DLC, se tiene un mejor comportamiento de las muestras recubiertas; basados en la diferencia que se percibe en las superficies de los dos sistemas se puede inferir que con mayor variación de topografía se produce mayor degradación, ya sea por el mecanismo de microabrasión o por los fenómenos corrosivos. Adicionalmente, en las pruebas de desgaste para ambos sistemas se observó una rugosidad promedio menor a la obtenida por los sistemas evaluados con fenómenos sinérgicos de corrosión- desgaste; esto se puede atribuir a que el material del pin que generó el desgaste está compuesto por hueso, que, a su vez, está conformado por colágeno, compuesto que puede generar una capa superficial de lípidos que protege la superficie del desgaste, pero que al aplicar la solución acuosa para generar el ensayo de corrosión esta capa no se presenta, incrementando el deterioro del material en los ensayos de corrosión-desgaste.

\section{Conclusiones}

Con las mediciones electroquímicas se observó una considerable protección del recubrimiento, equivalente a más de tres veces la resistencia total del sistema; este comportamiento se debe a la interfaz que existe entre el sustrato y la capa de DLC generada por el silicio, la cual permite una transición suave entre la unión de los dos sistemas. El valor de la resistencia a la polarización, de 18,21 kohms, indica un efecto protector que genera el sistema debido a su bajo coeficiente de fricción y buena resistencia a la corrosión. Los resultados de las curvas potenciodinámicas muestran que el acero 316LVM tiene un potencial de corrosión de -478 
$\mathrm{mV}$ hacia una zona catódica, lo cual genera un bajo desempeño electroquímico, generando disolución acelerada, porque la corriente tiene un incremento espontáneo; en contraste, el recubrimiento tiene un potencial de corrosión de $-81 \mathrm{mV}$ hacia la zona anódica, mejorando su comportamiento debido a la generación de una inercia química y un incremento de su resistencia a la corrosión.

La topografía del acero muestra un proceso de degradación cuando se expone a fenómenos de microabrasión y microabrasión-corrosión, el cual se representa en pérdida de material y generación de mayor heterogeneidad en la superficie, aumentando la rugosidad; se puede inferir que con mayor variación de topografía se produce mayor degradación, ya sea por procesos corrosivos o de desgaste, debido a que el recubrimiento DLC/Si protege el sistema mediante la eliminación de crestas en la superficie, pero sin eliminarlas en su totalidad.

\section{Agradecimientos}

A la Vicerrectoría de Investigaciones de la Universidad Militar Nueva Granada, por el apoyo financiero; proyecto ING-1775.

\section{REFERENCIAS}

[1] E. H. Mirza, S. F. Akbar y M. Asif, Evaluation of Anti-Corrosion Properties of DLC Coatings for Medical Devices. Kuala Lumpur, Malaysia: University of Malaya, Biomedical Engineering (ICoBE), 2012, International Conference.

[2] J.Sui, W.Cai,A.Liu,Z. Wang y L.Zhao, "Surface Characterization and Blood Compatibility of Diamond-Like Carbon (DLC) Films on the NiTi Alloys", Materials Transactions, vol. 47, no. 3, pp. 691-693, 2006. DOI: http://dx.doi. org/10.2320/matertrans.47.691.

[3] Y. Fu, H. Du y Q. Sun, "Interfacial structure, residual stress and adhesion of diamond coatings deposited on titanium", Thin Solid Films, vol. 424, pp. 107-114, 2003. DOI: http:// dx.doi.org/10.1016/S0040-6090(02)00908-2.

[4] J. Vetter, " 60 years of DLC coatings: Historical highlights and technical review of cathodic arc processes to synthesize various DLC types, and their evolution for industrial applications", Surface and Coatings Technology, vol.
257, pp. 213-240, 2014. DOI: http://dx.doi. org/10.1016/j.surfcoat.2014.08.017.

[5] D. Luo, V. Fridrici y P. Kapsa, "Relationships between the fretting wear behavior and the ball cratering resistance of solid lubricant coatings", Surface and Coatings Technology, vol. 204, no. 8, pp. 1259-1269, 2010. DOI: http://dx.doi. org/10.1016/j.surfcoat.2009.10.019.

[6] K. Shiba, Y. Ohgoe, K. Hirakuri1, J. Mizuno, S. Shoji, K. Ozeki y K. Sato, Hemocompatibility of DLC coating for blood analysis devices, Tokyo, Japan: School of Engineering and Graduate School of Engineering, Tokyo Denki University, Electronics Packaging (ICEP), 2014 International Conference.

[7] A. Gant, M. Gee y A. May, "Microabrasion of WC-Co hardmetals in corrosive media", Wear, vol. 256, no. 9-10, pp. 954-962, 2004. DOI: http://dx.doi.org/10.1016/j.wear.2003.09.003.

[8] L. Yang, A. Neville, A. Brown, P. Ransom y A. Morina, "Friction reduction mechanisms in boundary lubricated W-doped DLC coatings", Tribology International, vol. 70, pp. 2633, 2014. DOI: http://dx.doi.org/10.1016/j. triboint.2013.09.020.

[9] L. Bonetti, G. Capote, L. Santos y E. Corat, "Adhesion studies of diamond - like carbon films deposited on Ti6Al4V substrate with a silicon interlayer", Thin Solid Films, vol. 515, no. 1 , pp. $375-379,2006$. DOI: http://dx.doi. org/10.1016/j.tsf.2005.12.154.

[10] W. Aperador, G. Roa-Rodríguez y A. Mejía, "Characterization and Corrosion Behavior of Multilayer [TiAlN]nGrowthon AISI 316LVM Steel", Int. J. Electrochem. Sci., vol. 9, pp. 5025-5034, 2014.

[11] K. Lukaszkowicz, J. Sondor y K. Balin, "Characteristics of CrAlSiN + DLC coating deposited by lateral rotating cathode arc PVD and PACVD process", Applied Surface Science, vol. 312, pp. 126-133, 2014. DOI: http://dx.doi. org/10.1016/j.apsusc.2014.03.024.

[12] C. Martins, J. Moreira y J. Martins, "Corrosion in water supply pipe stainless steel 304 and a supply line of helium in stainless steel 316", Engineering Failure Analysis, vol. 39, pp. 6571, 2014. DOI: http://dx.doi.org/10.1016/j. engfailanal.2014.01.017.

[13] W. Aperador, G. Rodríguez y F. Franco, "Comportamiento de la corrosión de aleaciones 
de magnesio AZ31-B en ambiente marino, modificadas por el proceso de fricciónagitación", Rev. chil. ing., vol. 20, no. 1, pp. 119-125, 2012.

[14] V.Muthukumarany V.Selladurai, "Experimental Evaluaton of Corrosion and Hardness on AISI 316L Stainless Steel Implanted with Nitrogen and Argon Ions", Acta Mechanica Slovaca, vol. 14, no. 1, pp. 80-87, 2010. DOI: http://dx.doi. org/10.2478/v10147-011-0011-2.

[15] D. Sun, J. Wharton, R.J.K. Wood, L. Ma y W.M. Rainforth, "Microabrasion-corrosion of cast CoCrMo alloy in simulated body fluids", vol. 42, no. 1, pp. 99-110, 2009.

[16] C. Chandrasatheesh, J. Jayapriya, R. George y U. K. Mudali, "Detection and analysis of microbiologically influenced corrosion of 316
L stainless steel with electrochemical noise technique", Engineering Failure Analysis, vol. 42, pp. 133-142, 2014. DOI: http://dx.doi. org/10.1016/j.engfailanal.2014.04.002.

[17] G. Zambrano, H. Riascos y P. Prieto, "Multicapas de metal-cerámico-carbono tipo diamante (DLC): un camino para la obtención de recubrimientos superduros", Rev. Acad. Colomb. Cienc., vol. 27, no. 103, pp. 225-231, 2003.

[18] Y. Liua, D. Biana, Y. Wub, N. Li, K. Qiuc y Y. Zhenga, "Influence of biocompatible metal ions $(\mathrm{Ag}, \mathrm{Fe}, \mathrm{Y})$ on the surface chemistry, corrosion behavior and cytocompatibility of $\mathrm{Mg}-1 \mathrm{Ca}$ alloy treated with MEVVA", Colloids Surf B: Biointerfaces, pp. 99-107, 2015. DOI: http:// dx.doi.org/10.1016/j.colsurfb.2015.05.050. 